\title{
Diseño e implementación de un prototipo automatizado para el procesamiento de pastas y líquidos
}

\author{
(1) Edgar Ruiz Lizama \\ (2) Jorge Inche Mitma \\ (3) Alfonso Chung Pinzás \\ (4) Roberto Tello Yuen
}

\section{INTRODUCCIÓN}

\section{RESUMEN}

El artículo trata el siguiente problema: ¿Qué instrumentos físicos y virtuales interactuan en una Interface Hombre Máquina a fin automatizar un prototipo para el Procesamiento de Pastas y Líquidos?; El diseño incluye el dimensionado y la selección de dispositivos. La implementación considera la plataforma SYSTEM 302 para la supervisión y control de procesos; se obtuvo como resultado tangible un módulo automatizado cuya inversión fue devuelta mediante los cursos del CEMA.

Palabras Clave. Automatización, SCADA, proceso, sensores, producción.

DEsign AND Implementation OF A Prototype Automated For The PROCESSING OF PASTES AND LIQUIDS

\section{ABSTRACT}

The article focused the next problem: What physical and virtual instruments interact in an interface Human Machine in order to automate a prototype to the process of pastes and liquids?; the design includes the measured and the selection of devices. The implementation considers the SMAR'S SYSTEM 302 platform to the process supervision and control; As a result of this we have an automated module whose investment was returned by the CEMA's courses.

Key Words: Automation, SCADA, process, sensors, production.
La automatización de procesos es un tema de gran actualidad en diversas partes del mundo, la presentación de los resultados de la investigación en el presente artículo se debe a la necesidad de divulgar las posibilidades que ofrece la automatización no sólo a las grandes empresas sino también a procesos que pueden ser aplicados por las PYMEs del país. Por otro lado, la importancia de conocer los fundamentos de la automatización de procesos radica en que permitirá al lector tener una visión más amplia sobre que proceso o que parte del proceso poder automatizar.

El objetivo de la investigación es concretar la teoría del tema en un producto tangible a fin de demostrar su utilidad. La investigación se justifica debido a que se presentan algunas pautas importantes así como criterios técnicos para realizar los cambios en los procesos productivos, el estudio se limita a procesos de similares características al presentado en el artículo.

Cabe mencionar que el prototipo automatizado para el procesamiento de pastas y líquidos constituye el esfuerzo de docentes y alumnos así como un producto del Centro de Manufactura Avanzada (CEMA) de la Facultad de Ingeniería Industrial de la UNMSM.

\section{LAPIRÁMIDE DE LAAUTOMATIZACIÓN INDUSTRIAL}

Dentro de la industria existen distintos niveles de automatización, los cuales se agrupan dentro de la pirámide de la automatización; estos niveles son los siguientes:

\section{- Nivel 0: Tecnologías primarias}

En este nivel se agrupan las tecnologías tradicionales de producción; sobre todo aquellas que se basan en el trabajo manual; aunque en la actualidad esta tecnología tiende a ser reemplazada por la automatización, aún tiene vigencia en muchas PYMEs peruanas.

- Nivel 1: Control automatizado de procesos

Este nivel constituye un peldaño superior dentro de la automatización; es decir ya entran a reemplazar el trabajo humano

\footnotetext{
(1) Magíster en Informática. Profesor del Departamento de Ingeniería de Sistemas e Informática, UNMSM. E-mail:eruizl@unmsm.edu.pe

Magíster en Ciencias. Profesor del Departamento de Diseño y Tecnología Industrial, UNMSM. E-mail:jlinche@hotmail.com

Magíster en Ingeniería Industrial. Profesor del CEMA, UNMSM

E-mail: ramon_chung@yahoo.es

(4) Ingeniero Industrial. Profesor del Departamento de Ingeniería de Sistemas e Informática, UNMSM

E-mail: rtelloy@unmsm.edu.pe
} 
los sensores, actuadores y controladores, los cuales cumplen la función de control de procesos.

\section{- Nivel 2: Supervisión}

Este nivel está referido a la supervisión de los diversos procesos de producción los cuales se encuentran bajo el control automatizado de procesos; esto se realiza mediante software SCADA.

- Nivel 3: Ejecución

Este nivel está referido a la gerencia del proceso de fabricación; ejecutando las metas previstas por organismos superiores dentro de la organización.

\section{- Nivel 4: Empresa}

El nivel de empresa está relacionado con el planeamiento de la producción, generando las metas necesarias a ser ejecutadas por el nivel 3 .

- Nivel 5: Administración

Este nivel está asociado al rumbo de la organización; utiliza las herramientas de la planificación, estadística etc..

El estudio se ubica en el nivel 2; es decir el de supervisión.

\section{SISTEMA SCADA}

Un componente importante en todo proceso de automatización es el sistema SCADA, cuyo nombre proviene de las siglas de "Supervisory Control And Data Acquisition"; así pues este tipo de sistema se centra en el nivel de control de procesos y está basado en computadoras y software; entre las ventajas que más se le reconoce a este tipo de sistemas es que proporcionan un nivel de control más exacto del proceso, así como un historial del mismo; sin embargo la mayor utilidad que tienen estos sistemas radica en que se puede realizar el control a distancia, es decir no es necesario que la persona encargada esté en la planta sino puede hacerlo desde cualquier lugar e incluso país, esto gracias a Internet.

\section{DISEÑO DELPROTOTIPO}

El sistema prototipo realizará procesamiento de pastas y líquidos a la temperatura deseada, podrá ser controlado remotamente desde una computadora o también manualmente desde el panel de control; las variables a controlar serán la temperatura y el nivel; también se podrá controlar el giro del motor y la descarga del fluido.
Cuadro 1. Presión Versus Llenado

\begin{tabular}{|r|r|}
\hline PRESIÓN (Bares) & \multicolumn{1}{|c|}{ TIEMPO (s) } \\
\hline 1,00 & No registra salida \\
\hline 1,50 & 3,75 \\
\hline 2,00 & 3,48 \\
\hline 3,00 & 3,34 \\
\hline 4,50 & 3,10 \\
\hline 5,00 & 2,90 \\
\hline Valor Máximo $=6.00$ & 2,60 \\
\hline
\end{tabular}

Fuente: Elaboración propia

La presión adecuada fue determinada mediante la realización de pruebas de presión versus tiempo de llenado. Para un envase de 2 litros se obtuvieron los resultados mostrados en el Cuadro 1.

Las líneas de envasado automatizado consideran un tiempo de retención entre 2 a 4 segundos por consiguiente la presión de suministro de aire para la apertura y cierre de la válvula es de 3 bares. Se sugiere para la implementación un llenado con un sistema multivía de modo que para el tiempo de retención se pueda llenar 8 envases a la vez de 1/4 litro.

Con respecto a la variable Temperatura de Calentamiento; el mezclador multiusos permitirá un rango entre la temperatura ambiental y $80^{\circ} \mathrm{C}$. Este valor máximo se establece con el objetivo de evitar la evaporación del agua y solventes (alcoholes).

Sobre la variable nivel, el mezclador multiusos permite un rango entre 10 litros (nivel de ubicación de la válvula) y 180 litros (nivel con un $10 \%$ de espacio libre), para un mejor entendimiento véase el Cuadro 2.

\section{IMPLEMENTACIÓN DEL PROTOTIPO AUTOMATIZADO}

Para la implementación del módulo se partió de una mezcladora multiusos manual en donde se procedió a instalar sensores y transmisores de nivel y de temperatura, luego el controlador que es el sistema DFI 302, el cual fue programado con el software

\section{Cuadro 2. Rango de Nivel}

\begin{tabular}{|c|c|}
\hline VOLUMEN (Litros) & NIVEL (\%) \\
\hline 10 & 0 \\
\hline 30 & 11 \\
\hline 50 & 28 \\
\hline 180 & 100 \\
\hline
\end{tabular}

Fuente: Elaboración propia 
Figura 1. Prototipo terminado

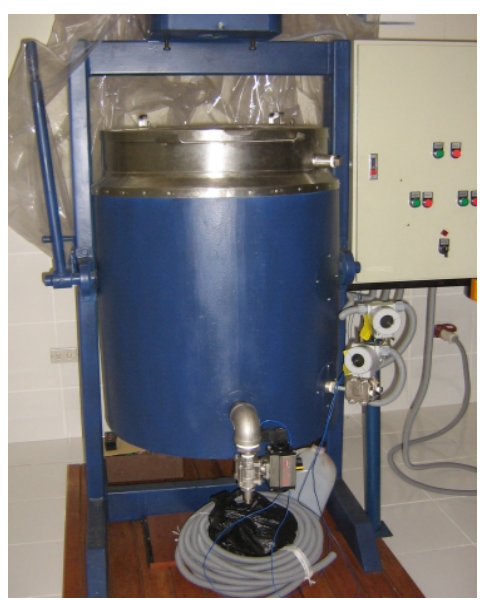

SYSCON ambos son parte de SYSTEM 302 y finalmente la válvula de descarga la cual trabaja con un compresor de aire aproximadamente a 3 bares. El resultado final del prototipo se presenta en la figura 1. A continuación se hará una breve explicación de los componentes.

\section{EI Controlador DFI 302 y el Software Syscon}

DFI 302 representa el Hardware de SYSTEM 302, su aplicación se centra en el manejo, control, monitoreo, mantenimiento y operación de una planta; tiene una arquitectura modular y puede ser expandible; la configuración básica consta de 4 módulos:

1. DF50 (Power Supply for Backplane): Este módulo es encarga de la fuente de alimentación del DFI 302.

2. DF51 (Powerful CPU Module): Este módulo es el que conecta el CPU con DFI 302

3. DF52 (Power Supply for Fieldbus): Este módulo energiza las redes de Fieldbus

4. DF53 (Power Supply Impedance for Fieldbus): Este modulo provee la impedancia necesaria a las redes Fieldbus

La figura 2 muestra dicha configuración. Originalmente el DFI 302 fue diseñado para trabajar con instrumentos Fieldbus, sin embargo muchas veces se requiere trabajar con instrumentos que no tienen comunicación Fieldbus, es así que DFI 302 puede ser expandible para abarcar estos instrumentos; en el caso del prototipo aparte de estos 4 módulos, se tiene 3 módulos más:

1. DF32: Cuenta con 8 entradas y 4 salidas en ambos casos trabajan con señales discretas, en
Figura 2. Configuración básica y tradicional de DFI 302

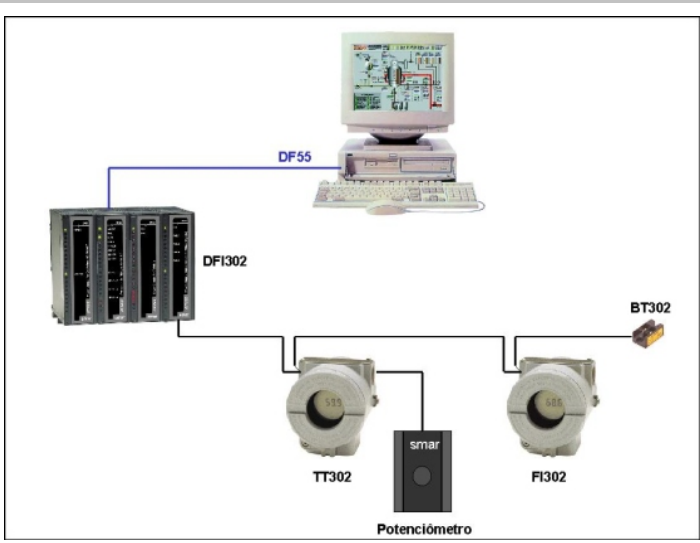

Fuente: Centro de Treinamento SMART (2002)

Manutenção Básica Fieldbus Foundation. Edición Digital. SMART. U.S.A.

este caso señales lógicas (verdadero o falso), en el prototipo las 4 salidas están conectadas a la válvula automática, al motor tanto para el sentido horario como antihorario y a la resistencia respectivamente.

2. Df44: Cuenta con 8 entradas análogas, de las cuales 2 están siendo usadas para los transmisores.

3. DF46: Cuenta con 4 salidas análogas, de las cuales una de ellas será destinada para la instalación de la faja transportadora con un variador de velocidad, en una segunda parte del proyecto.

La figura 3 presenta el DFI 302 del prototipo y la figura 4 presenta la configuración actual en el prototipo. En el software SYSCON se ha desarrollado una estrategia de control, el cual controla el giro del motor y el tiempo, así como la apertura o cierre de la válvula; el software de supervisión GaphWorX interviene como interface Hombre Máquina, el cual también forma parte de SYSTEM 302, la figura 5 muestra la pantalla de control.

Figura 3. Ubicación del DFI 302 en el prototipo

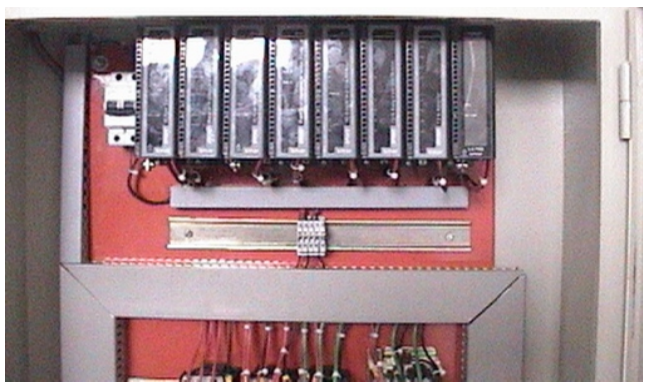


Figura 4. Configuración del Controlador DFI 302 en el prototipo
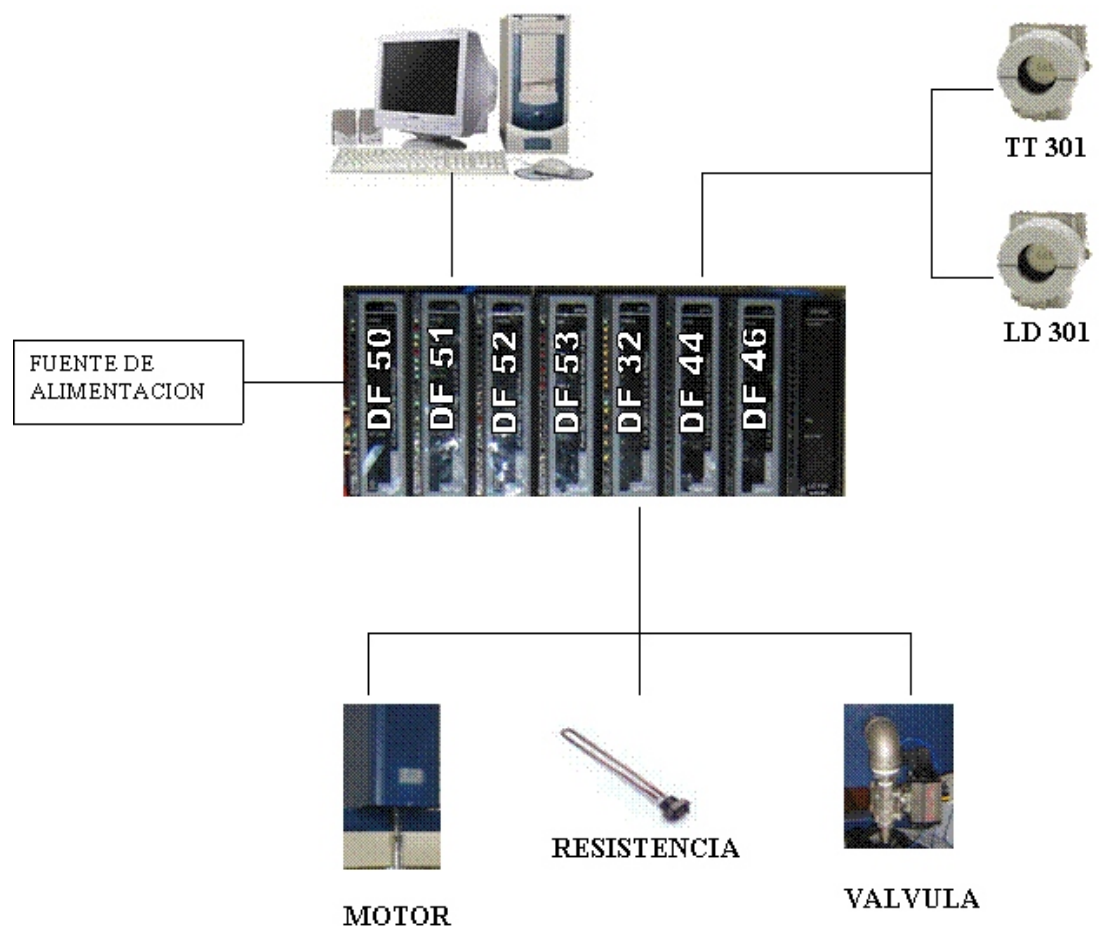

Figura 5. Pantalla del software de control del prototipo

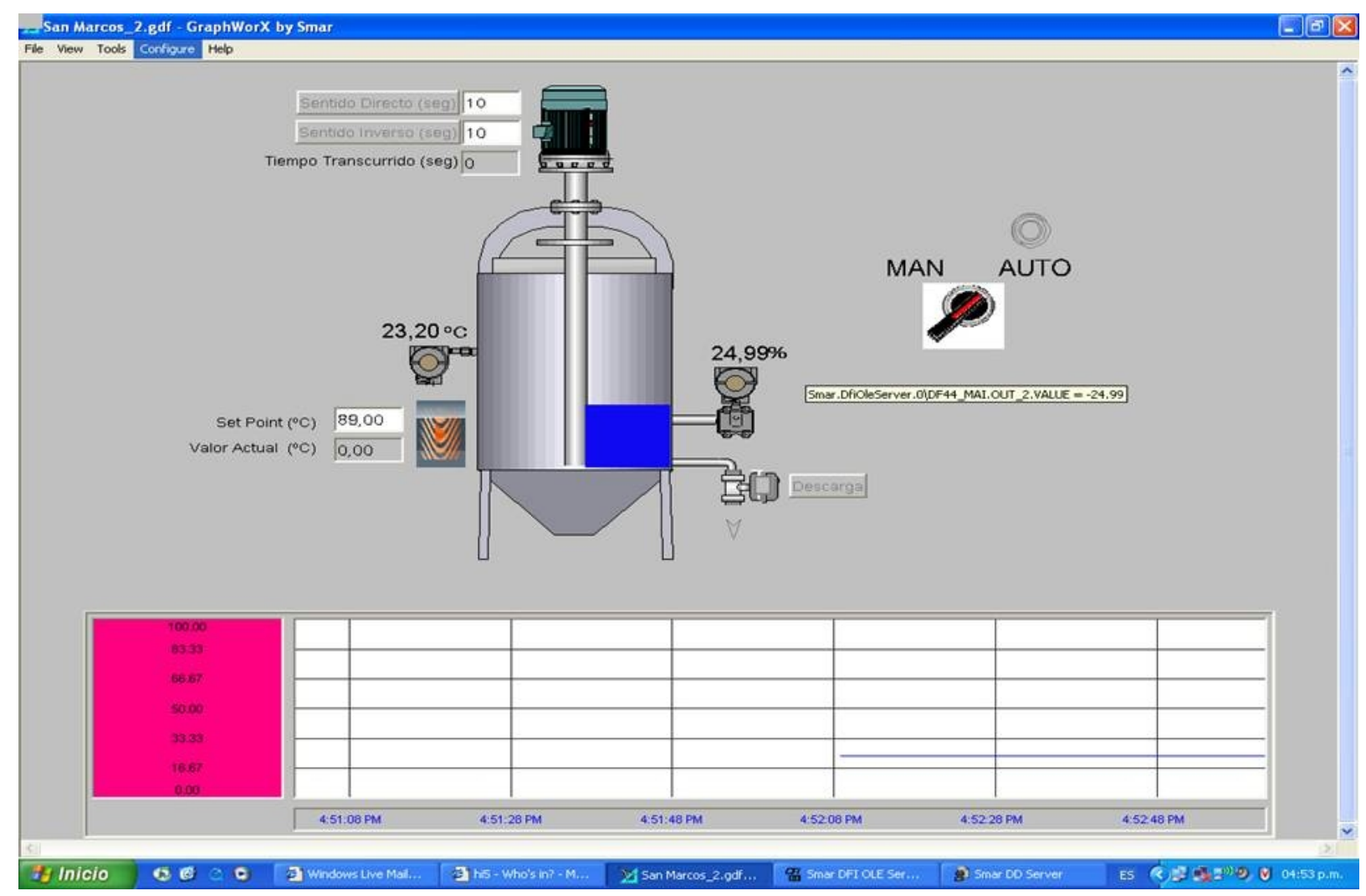


Figura 6. Ubicación de sensores y transmisores en el prototipo

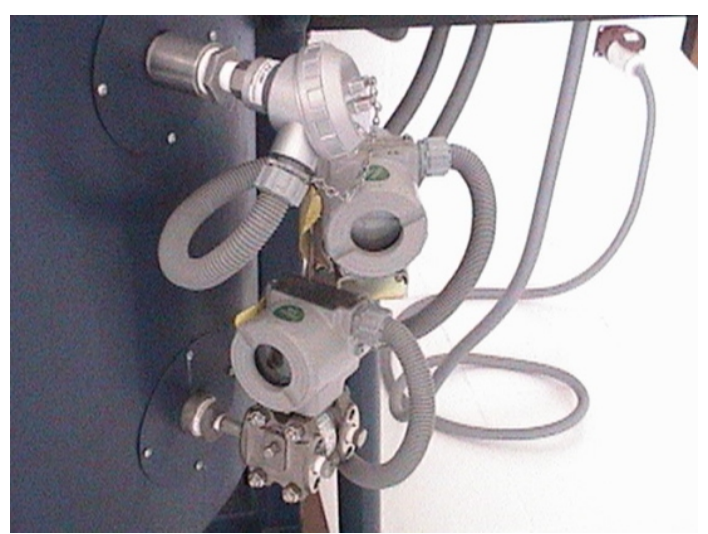

Sensores y Transmisores de Nivel y Temperatura Las variables de proceso elegidas para ser controladas son el Nivel y la Temperatura; para el primer caso se ha utilizado el sensor capacitivo y para el segundo caso un sensor PT 100.

Los transmisores son: el LD-301, el cual es un transmisor de diferencial de presión inteligente y programable, y tiene la ventaja de que puede ser programado y consultado a distancia en un terminal portátil; para el caso del proyecto se ha programado para trabajar como transmisor de nivel, sin embargo tiene diversas aplicaciones tales como: Cálculo de presión diferencial, medición de caudal, presión relativa, presión absoluta, nivel sanitario etc. La comunicación del Terminal al sensor se efectúa con el protocolo HART y del sensor al controlador mediante la señal de 4-20 mA; es por ese motivo que está conectado al módulo DF44 del DFI 302.

Para el caso de la variable temperatura se ha utilizado el transmisor TT-301, el cual trabaja con la casi totalidad de captadores de temperatura (entre ellos el PT100); similar al caso anterior este puede ser programado y consultado a distancia y se comunica mediante protocolo HART, se ubica en el módulo DF44 del DFI 302 junto con el sensor de nivel. La figura 6 ilustra la ubicación de los sensores y transmisores en el prototipo desarrollado.

\section{Protocolo HART}

Es el protocolo HART (Highway Addresable Remote Transducer) es otro componente del sistema, este protocolo es usado por gran parte de los instrumentos de campo y como se mencionó anteriormente, también es usado en el presente proyecto; HART utiliza el estándar de comunicación telefónica BELL 202; asimismo trabaja con salidas analógicas convencionales de 4-20 mA DC (usado por muchos instrumentos de campo) y a la vez con una salida
Figura 7: Superposicion de Señales dn dl Protocolo Hart

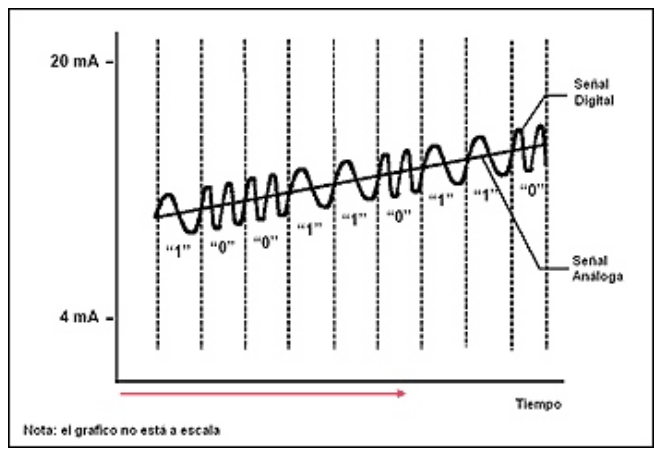

Fuente: Meichsner, K. (2006). El protocolo HART. http://www.emb.cl/electroindustria/articulo.mv?xid=95\&rank=1

digital de $1200 \mathrm{~Hz}$ y $2200 \mathrm{~Hz}$ (para los bits 0 y 1 respectivamente); es decir ambas señales están superpuestas mediante el principio de Cambio Codificado de Frecuencia o FSK, la figura 7 ilustra la superposición de las señales.

Entre las ventajas de este protocolo se tienen las siguientes:

1. Es de fácil instalación y puesta en marcha.

2. Es de fácil uso.

3. Es compatible con la mayoría de instrumentos de campo ya que trabaja con la señal análoga tradicional de 4-20 mA.

4. Permite transmitir varias medidas de un instrumento de campo.

\section{PRUEBA DEL PROTOTIPO: PRODUCCIÓN DE UN LOTE DE PASTA LIMPIA COMPUTADORAS}

Una vez instalado el prototipo, se inició la producción a modo de prueba, obteniéndose resultados satisfactorios, es decir un primer lote de producción del producto en cuestión (ver figura 8).

Figura 8. Primer lote de producción

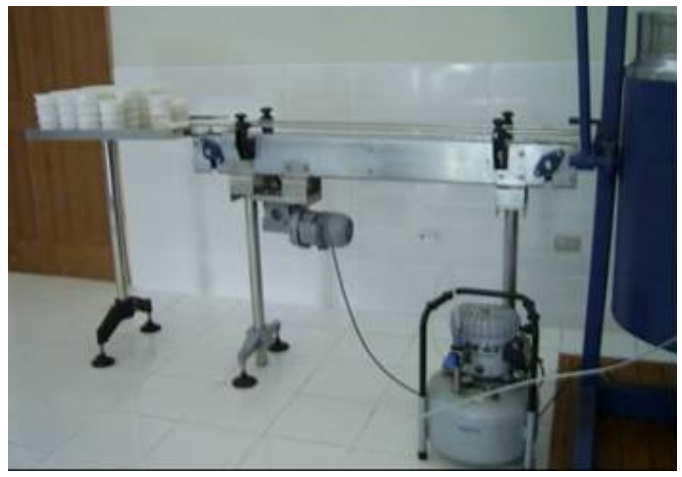




\section{PERSPECTIVAS DEL PROYECTO}

El proyecto ha sido finalizado y probado con éxito en una primera fase, sin embargo aún queda por ejecutar una segunda parte la cual se centra en un sistema mixto HART - FIELDBUS e incluye el diseño y la instalación de un transportador a usarse en el envasado del producto, es necesario instalar un sensor de presencia, calcular el tiempo de llenado que será el de parada de dicha faja entre otras variables; una vez finalizado esto, se podrá hacer la comparación de costos entre un proceso tradicional y otro totalmente automatizado.

\section{CONCLUSIONES}

El procesamiento del producto mejora con el prototipo automatizado debido a que las variables tanto de nivel como de temperatura se controlan mejor.

El esfuerzo del operario en la producción se reduce con el prototipo automatizado, debido a que todas las etapas que le siguen al llenado (incluyendo la descarga) se pueden hacer desde la computadora.

Debido a que se registran las variaciones de temperatura, el prototipo automatizado propor-ciona una mayor facilidad para realizar el análisis del comportamiento de esta variable.

La programación en el software SYSCON, mediante estrategia de programación, proporciona una mejor idea de la lógica del funcionamiento de los instrumentos y su conexión con el controlador.

\section{RECOMENDACIONES}

Realizar convenios para asesoría con los diferentes gremios de PYMEs a fin de difundir esta tecnología y su aplicación.

Realizar proyectos similares con otras propuestas de procesamiento industrial (secado, molienda, etc.) a fin de desarrollar prototipos automatizados y generar bases de datos con los resultados obtenidos.

Establecer líneas de investigación con equipos multidisciplinarios (docentes, estudiantes y colaboradores externos) de proyectos que involucren supervisión y control automatizado de procesos.

\section{REFERENCIAS BIBLIOGRÁFICAS}

1. Centro de Treinamento SMART (2002). Manutenção Básica Fieldbus Foundation. Edición Digital. SMART. U.S.A.

2. Desin Instruments. Ficha Técnica Sensor TT 301. Desin Instruments.

En: http://www.desin.com/

(Visitado: 25-10-06)

3. Desin Instruments. Ficha Técnica Sensor LD 301. Desin Instruments. Edición Digital,

En: http://www.desin.com/

(Visitado: 25-10-06)

4. Meichsner, K. (2006). El protocolo HART.

En:http://www.emb.cl/electroindustria/articulo.m $\mathrm{v}$ ? $\mathrm{xid}=95$ \& rank $=1$

(Visitado: 24-10-06)

5. Piñón, A. (2001). Tecnologías actuales de comunicación de las variables de campo en la industria de proceso. En:http://www.ceaifac.es/actividades/jornadas/XXII/documentos/A 03_IC.pdf

(Visitado: 24-10-06)

6. SMART. (2005). Fieldbus Universal Bridge User's Manual. SMART. U.S.A.

7. SMART (2004). SYSCON System Configurator Installation and Operation Manual. Edición Digital. SMART. U.S.A.

3. SMART (2004). GraphWorX User's Guide. Edición Digital. SMART. U.S.A. 